\title{
GEOMETRIC CALIBRATION OF ZIYUAN-3 THREE-LINE CAMERAS COMBINING GROUND CONTROL POINTS AND LINES
}

\author{
Jinshan Cao ${ }^{\text {a }}$, Xiuxiao Yuan ${ }^{\text {a, b, *, Jianya Gong }}{ }^{\text {a, b }}$ \\ ${ }^{a}$ Collaborative Innovation Center of Geospatial Technology, Wuhan 430079, China - (caojs, yuanxx, gongjy)@whu.edu.cn \\ ${ }^{\mathrm{b}}$ School of Remote Sensing and Information Engineering, Wuhan University, Wuhan 430079, China
}

Commission I, WG I/3

KEY WORDS: Geometric calibration, three-line cameras, ground control lines, direct georeferencing, orientation parameters, Ziyuan-3, block adjustment

\begin{abstract}
:
Due to the large biases between the laboratory-calibrated values of the orientation parameters and their in-orbit true values, the initial direct georeferencing accuracy of the Ziyuan-3 (ZY-3) three-line camera (TLC) images can only reach the kilometre level. In this paper, a point-based geometric calibration model of the ZY-3 TLCs is firstly established by using the collinearity constraint, and then a line-based geometric calibration model is established by using the coplanarity constraint. With the help of both the point-based and the line-based models, a feasible in-orbit geometric calibration approach for the ZY-3 TLCs combining ground control points (GCPs) and ground control lines (GCLs) is presented. Experimental results show that like GCPs, GCLs can also provide effective ground control information for the geometric calibration of the ZY-3 TLCs. The calibration accuracy of the look angles of charge-coupled device (CCD) detectors achieved by using the presented approach reached up to about 1.0". After the geometric calibration, the direct georeferencing accuracy of the ZY-3 TLC images without ground controls was significantly improved from the kilometre level to better than $11 \mathrm{~m}$ in planimetry and $9 \mathrm{~m}$ in height. A more satisfactory georeferencing accuracy of better than $3.5 \mathrm{~m}$ in planimetry and $3.0 \mathrm{~m}$ in height was achieved after the block adjustment with four GCPs.
\end{abstract}

\section{INTRODUCTION}

In-orbit geometric calibration is an indispensable procedure in the wide applications of high-resolution satellite (HRS) imagery. For the majority of HRS sensors, such as IKONOS, SPOT-5, OrbView-3, ALOS, GeoEye-1, and PLEIADES sensors, the geometric calibration was all performed, as referred in (Crespi et al., 2010; De Lussy et al., 2012; Gachet, 2004; Grodecki and Lute, 2005; Mulama, 2004; Radhadevi, 2011). Although the geometric calibration approaches may differ from each other due to the physical structures of different HRS sensors, the objectives of the geometric calibration are same, i.e., improving the georeferencing accuracy of HRS imagery by periodically calibrating the orientation parameters of HRS sensors.

The Ziyuan-3 (ZY-3) satellite is the first civilian high-resolution stereo mapping satellite of China. It is equipped with three-line cameras (TLCs) looking forward (FWD), nadir (NAD), and backward (BWD), respectively. The ground sample distance (GSD) of the NAD camera is about $2.1 \mathrm{~m}$, and the GSD of both the FWD and BWD cameras is about $3.5 \mathrm{~m}$. The swath width of the TLCs is all about $51 \mathrm{~km}$. The base-to-height ratio formed by the FWD and BWD cameras is about 0.8. Similarly, serval feasible and effective geometric calibration approaches for the ZY-3 TLCs were developed, as referred in (Cao et al., 2015; Chen et al., 2015; Wang et al., 2014; G. Zhang et al., 2014; Y. Zhang et al., 2014). With the help of the above geometric calibration approaches, the direct georeferencing accuracy of the ZY-3 TLC images without ground controls was significantly improved. However, the above approaches took only ground control points (GCPs) as control information. The GCPs were mainly surveyed in the field or extracted from the reference digital orthophoto map (DOM) and digital elevation model (DEM). When sufficient evenly distributed GCPs are not available due to the lack of clear point features in the field or the large date interval between the ZY-3 TLC images and the DOM and DEM, it will be very difficult to periodically perform the geometric calibration of the ZY-3 TLCs using only GCPs. Therefore, taking full advantage of other ground control information to perform the geometric calibration may be a better and more feasible solution.

Compared with point features, linear features can still be used in photogrammetric activities, such as relative orientation, exterior orientation, block adjustment, and image registration, even without a complete match between the lines in image space and the lines in object space (Habib and Alruzouq, 2004; Karjalainen et al., 2006; Marcato Junior and Tommaselli, 2013; Tommaselli and Medeiros, 2010; Zhang et al., 2008; Zhang et al., 2011). Taking full advantage of linear features, a feasible inorbit geometric calibration approach for the ZY-3 TLCs combining GCPs and ground control lines (GCLs) is presented in this paper. For the presented approach, both the GCPs and GCLs can be taken as ground controls. When it is difficult to obtain sufficient GCPs, GCLs can be used to substitute for the absent GCPs, and the geometric calibration of the ZY-3 TLCs can be realized by using the presented approach. This paper is organised in four sections. In the next section, both the pointbased and line-based geometric calibration models of the ZY-3 TLCs are established, and the solutions of the geometric calibration are introduced briefly. In the following section, three datasets of the ZY-3 TLC images as well as the GCPs and GCLs

\footnotetext{
* Corresponding author
} 
are used to validate the feasibility and effectiveness of the presented approach. Finally, the conclusions of this paper are provided.

\section{GEOMETRIC CALIBRATION MODELS AND SOLUTIONS}

When we perform the geometric calibration of the ZY-3 TLCs combining GCPs and GCLs, we should establish the pointbased and line-based geometric calibration models separately. In this paper, the point-based geometric calibration model established by Cao et al. (2015) is employed and used. Based on the point-based geometric calibration model, the line-based geometric calibration model of the ZY-3 TLCs is established.

\subsection{Point-based Geometric Calibration Model}

With the help of the measured satellite positions and attitudes and the laboratory-calibrated orientation parameters, we can firstly establish the physical sensor model of the ZY-3 TLCs as follows:

$$
\left[\begin{array}{c}
X_{P} \\
Y_{P} \\
Z_{P}
\end{array}\right]_{\mathrm{WGS} 84}=\left[\begin{array}{c}
X_{S} \\
Y_{S} \\
Z_{S}
\end{array}\right]_{\mathrm{WGS} 84}+m \boldsymbol{R}_{\mathrm{J} 2000}^{\mathrm{WGS} 84} \boldsymbol{R}_{\mathrm{Star}}^{\mathrm{J2000}}\left(\boldsymbol{R}_{\mathrm{Star}}^{\mathrm{Body}}\right)^{\mathrm{T}} \boldsymbol{R}_{\text {Camera }}^{\mathrm{Body}}\left[\begin{array}{c}
\tan \left(\psi_{y}\right) \\
\tan \left(\psi_{x}\right) \\
-1
\end{array}\right] \cdot f
$$

where, $\left(X_{P}, Y_{P}, Z_{P}\right)_{\mathrm{WGS} 84}^{\mathrm{T}}$ and $\left(X_{S}, Y_{S}, Z_{S}\right)_{\mathrm{WGS} 84}^{\mathrm{T}}$ represent the WGS84 coordinates of the ground point $P$ and the satellite position $S$, respectively; $m$ is the scale factor; $\boldsymbol{R}_{\mathrm{J} 2000}^{\mathrm{WGS} 4}$ represents the rotation matrix from the Julian-year-2000 (J2000) coordinate system to the WGS84 coordinate system; $\boldsymbol{R}_{\text {Star }}^{\mathrm{J} 2000}$ and $\boldsymbol{R}_{\text {Star }}^{\text {Body }}$ are the rotation matrices from the attitude determination reference coordinate system to the J2000 coordinate system and to the satellite-body coordinate system, respectively; $\boldsymbol{R}_{\text {Canera }}^{\text {Body }}$ represents the rotation matrix from the camera coordinate system to the satellite-body coordinate system; $\left(\psi_{y}, \psi_{x}\right)$ is the CCD-detector look angles in the camera coordinate system; and $f$ is the focal length.

Due to the high acceleration during the satellite's launch, the variation of spatial environments, and the degeneration of the equipped optical instruments, the orientation parameters $\left(\psi_{y}, \psi_{x}\right)$, $f, \boldsymbol{R}_{\text {Camera }}^{\text {Body }}$ and $\boldsymbol{R}_{\text {Star }}^{\text {Body }}$ in Equation 1 may change more or less when the satellite operates in the orbit. In theory, we should calibrate these orientation parameters separately. However, these orientation parameters have very strong correlations between each other, so that it is very difficult to separate them. In order to avoid the strong correlations in the geometric calibration of the ZY-3 TLCs, Cao et al. (2015) used the CCD-detector look angles in the attitude determination reference coordinate system to describe the comprehensive effects of the orientation parameters $\left(\psi_{y}, \psi_{x}\right), f, \boldsymbol{R}_{\text {Camera }}^{\text {Body }}$, and $\boldsymbol{R}_{\text {Star }}^{\text {Body }}$ on the georeferencing of the ZY-3 TLC images and established a simple and valid physical sensor model as follows:

$$
\left[\begin{array}{c}
X_{P} \\
Y_{P} \\
Z_{P}
\end{array}\right]_{\mathrm{WGS} 84}=\left[\begin{array}{c}
X_{S} \\
Y_{S} \\
Z_{S}
\end{array}\right]_{\mathrm{WGS} 84}+\lambda \boldsymbol{R}_{\mathrm{J} 2000}^{\mathrm{WGS} 84} \boldsymbol{R}_{\mathrm{Star}}^{\mathrm{J} 2000}\left[\begin{array}{c}
\tan \left(\psi_{y}^{\prime}\right) \\
\tan \left(\psi_{x}^{\prime}\right) \\
-1
\end{array}\right]
$$

where, $\lambda$ is the scale factor and $\left(\psi_{y}^{\prime}, \psi_{x}^{\prime}\right)$ represents the CCDdetector look angles in the attitude determination reference coordinate system.

According to Equation 2, the point-based geometric calibration model of the ZY-3 TLCs can be established as follows:

$$
\left[\begin{array}{c}
\tan \left(\psi_{y}^{\prime}\right) \\
\tan \left(\psi_{x}^{\prime}\right) \\
-1
\end{array}\right]=\frac{1}{\lambda}\left(\boldsymbol{R}_{\mathrm{J} 2000}^{\mathrm{WGS} 84} \boldsymbol{R}_{\mathrm{Star}}^{\mathrm{J} 2000}\right)^{\mathrm{T}}\left\{\left[\begin{array}{c}
X_{P} \\
Y_{P} \\
Z_{P}
\end{array}\right]_{\mathrm{WGS} 84}-\left[\begin{array}{c}
X_{S} \\
Y_{S} \\
Z_{S}
\end{array}\right]_{\mathrm{WGS} 84}\right\}
$$

\subsection{Line-based Geometric Calibration Model}

As shown in Figure 1, the line $L$ in object space is a GCL determined by the points $P_{1}$ and $P_{2}$. The line $l$ in image space is the corresponding line of the line $L$. The points $t_{1}$ and $t_{2}$ are the end points of the line $l$, and the point $p$ is an arbitrary point on the line $l$. The point $S$ is the instantaneous projection centre corresponding to the point $p$. The coordinate system $S-x y z$ is an auxiliary image-space coordinate system parallel to the objectspace coordinate system that refers to the WGS84 coordinate system in this paper.

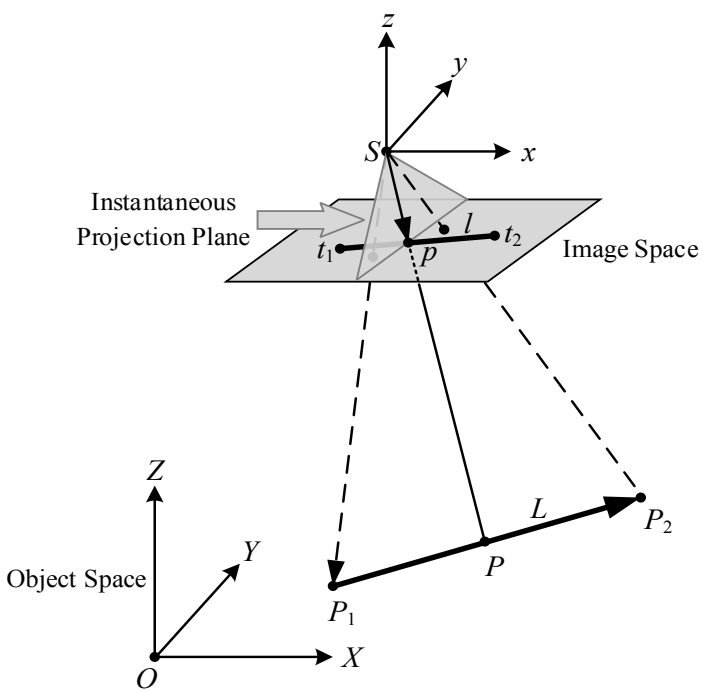

Figure 1. Imaging sketch map of linear features.

Unlike the traditional frame cameras, the ZY-3 FWD, NAD, and BWD cameras are all linear array cameras. It means that the line $L$ in object space and the corresponding line $l$ in image space cannot always lie in a same projection plane. However, we can see from Figure 1 that the ground point $P$ corresponding to the image point $p$ must always lie on the line $L$ in object space. That's to say, the projection line $S p$, the ground line $L$, and the line $S P_{1}$ must always lie in a same plane. Therefore, according to this geometric constraint, we can establish a coplanarity equation in the WGS84 coordinate system as follows:

$$
\overrightarrow{S P_{1}} \times \overrightarrow{P_{1} P_{2}} \cdot \overrightarrow{S p}=0
$$

Suppose that the WGS84 coordinates of the points $P_{1}$ and $P_{2}$ are $\left(X_{1}, Y_{1}, Z_{1}\right)$ and $\left(X_{2}, Y_{2}, Z_{2}\right)$, respectively. Accordingly, we can get

$$
\overrightarrow{S P_{1}} \times \overrightarrow{P_{1} P_{2}}=\left[\begin{array}{l}
d_{1} \\
d_{2} \\
d_{3}
\end{array}\right]=\left[\begin{array}{c}
\left(Y_{2}-Y_{1}\right)\left(Z_{1}-Z_{S}\right)-\left(Z_{2}-Z_{1}\right)\left(Y_{1}-Y_{S}\right) \\
\left(Z_{2}-Z_{1}\right)\left(X_{1}-X_{S}\right)-\left(X_{2}-X_{1}\right)\left(Z_{1}-Z_{S}\right) \\
\left(X_{2}-X_{1}\right)\left(Y_{1}-Y_{S}\right)-\left(Y_{2}-Y_{1}\right)\left(X_{1}-X_{S}\right)
\end{array}\right]
$$

Suppose that in the attitude determination reference coordinate system, the CCD-detector look angles corresponding to the image point $p$ are $\left(\psi_{y}^{\prime}, \psi_{x}^{\prime}\right)$. According to Equation 2, we can get 


$$
\overrightarrow{S p}=\lambda\left[\begin{array}{lll}
r_{11} & r_{12} & r_{13} \\
r_{21} & r_{22} & r_{23} \\
r_{31} & r_{32} & r_{33}
\end{array}\right]\left[\begin{array}{c}
\tan \left(\psi_{y}^{\prime}\right) \\
\tan \left(\psi_{x}^{\prime}\right) \\
-1
\end{array}\right]
$$

where, $r_{i j}(i=1,2,3 ; j=1,2,3)$ is the matrix element of the matrix $\boldsymbol{R}=\boldsymbol{R}_{\mathrm{J} 2000}^{\mathrm{WGSS4}} \boldsymbol{R}_{\mathrm{Star}}^{\mathrm{I2000}}$.

Substituting Equations 5 and 6 into Equation 4, we can then establish the line-based geometric calibration model of the ZY3 TLCs as follows:

$$
\begin{gathered}
\left(r_{11} d_{1}+r_{21} d_{2}+r_{31} d_{3}\right) \tan \left(\psi_{y}^{\prime}\right)+\left(r_{12} d_{1}+r_{22} d_{2}+r_{32} d_{3}\right) \tan \left(\psi_{x}^{\prime}\right) \\
-\left(r_{13} d_{1}+r_{23} d_{2}+r_{33} d_{3}\right)=0
\end{gathered}
$$

It is noted that the image point $p$ is an arbitrary point on the line $l$ rather than the corresponding image point of the ground point $P_{1}$ or $P_{2}$. That's to say, a complete match between the line $l$ in image space and the line $L$ in object space is unnecessary in the geometric calibration of the ZY-3 TLCs, which can provide useful conveniences in surveying GCLs in the field or extracting GCLs from the reference DOM and DEM.

\subsection{Simultaneous Adjustment}

Comparing Equation 7 with Equation 3, we can find that the unknown calibration parameters are only the CCD-detector look angles in the attitude determination reference coordinate system, no matter GCPs or GCLs are taken as ground controls in the geometric calibration of the ZY-3 TLCs. It demonstrates that from the point of view of calibration parameters, performing the geometric calibration combining GCPs and GCLs is feasible.

The ZY-3 NAD camera has 24530 equivalent CCD detectors, and both the FWD and BWD cameras have 16300 equivalent CCD detectors on the focal plane. It is very difficult and even impossible to calibrate the look angles of each CCD detector, since a very large number of ground controls are necessary. For this reason, the look-angle model in Cao et al. (2015) is used in the actual procedure of the geometric calibration of the $\mathrm{ZY}-3$ TLCs. The mathematical expression of the model is as follows:

$$
\left\{\begin{array}{l}
\psi_{y}^{\prime}=a_{0}+a_{1} N+a_{2} N^{2}+a_{3} N^{3} \\
\psi_{x}^{\prime}=b_{0}+b_{1} N+b_{2} N^{2}+b_{3} N^{3}
\end{array}\right.
$$

where, $N$ is the CCD-detector number; $\left(a_{0}, a_{1}, a_{2}, a_{3}, b_{0}, b_{1}, b_{2}, b_{3}\right)$ are the coefficients of the look-angle model.

Therefore, when we perform the geometric calibration of the ZY-3 TLCs combining GCPs and GCLs, only the coefficients of the look-angle model are actually unknowns. The main procedures of the geometric calibration are as follows:

(1) For each GCP, according to Equations 3 and 8, two error equations are established by using the image-space and objectspace coordinates of the GCP.

(2) For each GCL, according to Equations 7 and 8, two error equations are established by using the image-space and objectspace coordinates of the end points of the GCL.

(3) According to the above error equations relating to both the GCPs and GCLs, the coefficients of the look-angle model are solved by using the least-squares adjustment.

(4) According to Equation 8, the look angles of each CCD detector are calculated.

\section{RESULTS AND ANALYSIS}

\subsection{Study Areas and Datasets}

In this study, two ZY-3 datasets covering Songshan area and Taiyuan area, respectively, in China and one ZY-3 dataset covering Bellegarde area in France were tested. Each dataset has three images in the FWD, NAD, and BWD view, respectively. The general characteristics of the ZY-3 datasets are depicted in Table 1. Figure 2 shows the distribution of the GCPs and GCLs of each dataset.

\begin{tabular}{|c|c|c|c|}
\hline Characteristics & Dataset 1 & Dataset 2 & Dataset 3 \\
\hline $\begin{array}{c}\text { Geographic area } \\
\text { of images }\end{array}$ & $\begin{array}{c}\text { Songshan, } \\
\text { China }\end{array}$ & $\begin{array}{c}\text { Taiyuan, } \\
\text { China }\end{array}$ & $\begin{array}{c}\text { Bellegarde, } \\
\text { France }\end{array}$ \\
\hline Acquisition date & $\begin{array}{c}\text { February 3, } \\
2012\end{array}$ & $\begin{array}{c}\text { March 13, } \\
2012\end{array}$ & $\begin{array}{c}\text { February 29, } \\
2012\end{array}$ \\
\hline Terrain relief & 86 to & 743 to & 54 to \\
$1130 \mathrm{~m}$ & $1545 \mathrm{~m}$ & $245 \mathrm{~m}$ \\
\hline Number of GCLs & 10 & 0 & 0 \\
\hline Number of GCPs & 48 & 17 & 9 \\
\hline
\end{tabular}

Table 1. General characteristics of the ZY-3 datasets.
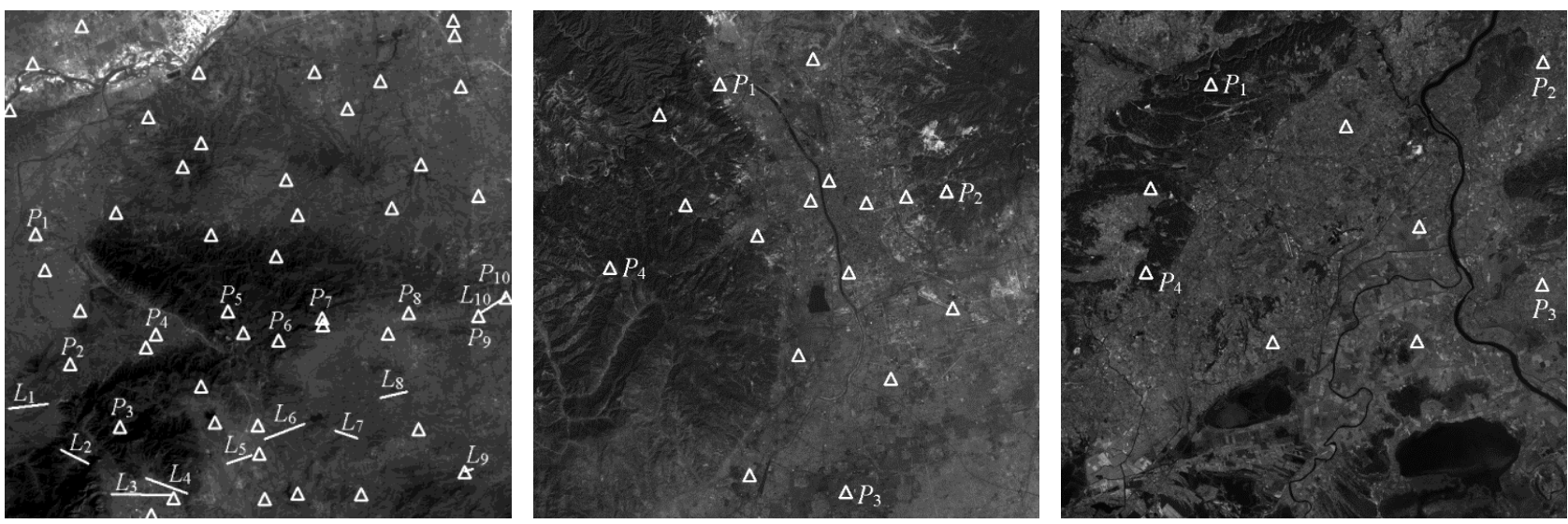

Figure 2. Distribution of the GCPs and GCLs in (a) dataset 1 (Songshan), (b) dataset 2 (Taiyuan), and (c) dataset 3 (Bellegarde). 
In the following experiments, the dataset 1 was used to calibrate the CCD-detector look angles of the ZY-3 TLCs. The datasets 2 and 3 were used to validate the georeferencing accuracy of the ZY-3 TLC images based on the calibrated CCD-detector look angles. The object-space coordinates of both the GCPs and GCLs in the dataset 1 were measured manually from the reference DOM and DEM. The GSD of the DOM and DEM is $0.2 \mathrm{~m}$ and $1.0 \mathrm{~m}$, respectively. The planimetric accuracy of the DOM is about $1.0 \mathrm{~m}$, and the height accuracy of the DEM is about $2.0 \mathrm{~m}$. The object-space coordinates of the GCPs in the datasets 2 and 3 were surveyed in the field, and both the planimetric and height accuracy is about $0.1 \mathrm{~m}$. The imagespace coordinates of both the GCPs and GCLs in the three datasets were all measured manually, and the measurement accuracy is about 0.5 pixel. In the dataset 1 , the measured GCLs are mainly the edges of rivers and roads. It is noted that when we measured the GCLs, the end points of the image line $l$ are unnecessary to correspond with the end points of the line $L$ in the reference DOM and DEM.

\subsection{Calibration Accuracy Analysis}

In order to evaluate the feasibility of calibrating the CCDdetector look angles of the ZY-3 TLCs combining GCPs and
GCLs, six ground control layouts, i.e., layout P10 with ten GCPs, layout P8L2 with eight GCPs and two GCLs, layout P6L4 with six GCPs and four GCLs, layout P4L6 with four GCPs and six GCLs, layout P2L8 with two GCPs and eight GCLs, and layout L10 with ten GCLs were designed and tested. For each ground control layout, the selected GCPs and GCLs in the dataset 1 distributed evenly in the across-track direction of the image-covered area, as shown in Figure $2 \mathrm{a}$ and listed in Table 2. The remaining GCPs were considered as independent check points (ICPs). Under each ground control layout, the CCD-detector look angles of the FWD, the NAD, and the BWD camera were calibrated separately according to the calibration procedure in the section 2.3 .

For the ZY-3 TLCs, in order to assess the calibration accuracy, the look angles of the CCD detectors corresponding to the ICPs were firstly calculated according to Equation 3 and considered as true values. After that, the root mean square error (RMSE) of the residual errors between the calibrated and the true CCDdetector look angles was calculated and listed in Table 2 .

\begin{tabular}{|c|c|c|c|c|c|}
\hline \multirow{2}{*}{ Camera } & \multirow{2}{*}{ Layout } & \multirow{2}{*}{ GCP and GCL Number } & \multirow{2}{*}{ Number of ICPs } & \multicolumn{2}{|c|}{ RMSE (") } \\
\hline & & & & $\psi_{y}^{\prime}$ & $\psi_{x}^{\prime}$ \\
\hline \multirow{6}{*}{ FWD } & $\mathrm{P} 10$ & $P_{1}$ to $P_{10}$ & 38 & 0.977 & 0.984 \\
\hline & P8L2 & $P_{1}$ to $P_{3}, L_{4}, P_{5}$ to $P_{7}, L_{8}, P_{9}, P_{10}$ & 40 & 1.092 & 1.098 \\
\hline & P6L4 & $P_{1}$ to $P_{3}, L_{4}, P_{5}, L_{6}, P_{7}, L_{8}, P_{9}, L_{10}$ & 42 & 1.092 & 1.104 \\
\hline & P4L6 & $L_{1}, P_{2}, P_{3}, L_{4}$ to $L_{6}, P_{7}, L_{8}, P_{9}, L_{10}$ & 44 & 1.051 & 0.907 \\
\hline & P2L8 & $L_{1}, L_{2}, P_{3}, L_{4}$ to $L_{6}, P_{7}, L_{8}$ to $L_{10}$ & 46 & 0.941 & 1.008 \\
\hline & L10 & $L_{1}$ to $L_{10}$ & 48 & 0.949 & 1.194 \\
\hline \multirow{6}{*}{ NAD } & $\mathrm{P} 10$ & $P_{1}$ to $P_{10}$ & 38 & 0.806 & 0.625 \\
\hline & P8L2 & $P_{1}$ to $P_{3}, L_{4}, P_{5}$ to $P_{7}, L_{8}, P_{9}, P_{10}$ & 40 & 0.868 & 0.622 \\
\hline & P6L4 & $P_{1}$ to $P_{3}, L_{4}, P_{5}, L_{6}, P_{7}, L_{8}, P_{9}, L_{10}$ & 42 & 0.797 & 0.602 \\
\hline & P4L6 & $L_{1}, P_{2}, P_{3}, L_{4}$ to $L_{6}, P_{7}, L_{8}, P_{9}, L_{10}$ & 44 & 0.669 & 0.728 \\
\hline & P2L8 & $L_{1}, L_{2}, P_{3}, L_{4}$ to $L_{6}, P_{7}, L_{8}$ to $L_{10}$ & 46 & 0.666 & 0.843 \\
\hline & L10 & $L_{1}$ to $L_{10}$ & 48 & 0.837 & 0.708 \\
\hline \multirow{6}{*}{ BWD } & $\mathrm{P} 10$ & $P_{1}$ to $P_{10}$ & 38 & 0.952 & 1.125 \\
\hline & P8L2 & $P_{1}$ to $P_{3}, L_{4}, P_{5}$ to $P_{7}, L_{8}, P_{9}, P_{10}$ & 40 & 1.171 & 1.072 \\
\hline & P6L4 & $P_{1}$ to $P_{3}, L_{4}, P_{5}, L_{6}, P_{7}, L_{8}, P_{9}, L_{10}$ & 42 & 1.138 & 1.162 \\
\hline & P4L6 & $L_{1}, P_{2}, P_{3}, L_{4}$ to $L_{6}, P_{7}, L_{8}, P_{9}, L_{10}$ & 44 & 1.036 & 1.178 \\
\hline & P2L8 & $L_{1}, L_{2}, P_{3}, L_{4}$ to $L_{6}, P_{7}, L_{8}$ to $L_{10}$ & 46 & 1.068 & 1.180 \\
\hline & L10 & $L_{1}$ to $L_{10}$ & 48 & 1.059 & 1.046 \\
\hline
\end{tabular}

Table 2. Calibration accuracy of the CCD-detector look angles of the ZY-3 TLCs in the dataset 1.

From the results in Table 2, we can see that using only GCPs in the layout P10, the calibration accuracy of the CCD-detector look angles of the FWD, NAD, and BWD cameras can reach about 1.0". As the number of the GCPs decreases and the number of the GCLs increases gradually, the calibration accuracy stay almost the same as that achieved by using only GCPs. Especially, when ten GCPs in the layout P10 were totally replaced by ten GCLs in the layout L10, the largest difference between the calibration accuracies achieved by using the layouts P10 and L10 is only 0.21". It demonstrates that like GCPs, GCLs can also provide sufficient and effective ground control information for the geometric calibration of the ZY-3 TLCs. In the geometric calibration, substituting GCLs for GCPs has almost no loss of the calibration accuracy of the CCD-detector look angles. Hence, when it is difficult to obtain sufficient evenly distributed GCPs, GCLs can be taken as an effective substitute for the absent GCPs.

\subsection{Georeferencing Accuracy Validation Based on the Calibrated Parameters}

For the geometric calibration of the ZY-3 TLCs, a more concerned topic is the geometric performance of the calibrated CCD-detector look angles in the georeferencing of the ZY-3 TLC images covering other areas. For this reason, in order to further evaluate the feasibility of the geometric calibration approach combining GCPs and GCLs, the direct georeferencing, i.e., spatial intersection, of the ZY-3 TLC images in the datasets 2 and 3 was firstly performed by taking the look angles calibrated by using each layout in the dataset 1 as input orientation parameters. Then, the block adjustment of the ZY-3 TLC images with four GCPs was performed.

The direct georeferencing of the datasets 2 and 3 was performed according to Equation 2, and the georeferencing accuracy is 
listed in Tables 3 and 4. Meanwhile, for convenient comparison, the initial direct georeferencing accuracy without the geometric calibration achieved according to Equation 1 is also listed as the layout $\mathrm{P} 0$ in Tables 3 and 4.

\begin{tabular}{|c|c|r|r|r|r|}
\hline \multirow{2}{*}{ Layout } & Number & \multicolumn{4}{|c|}{ RMSE (m) } \\
\cline { 3 - 6 } & of ICPs & North & East & Planimetry & Height \\
\hline P0 & 17 & 664.958 & 1021.741 & 1219.067 & 393.451 \\
\hline P10 & 17 & 4.336 & 9.240 & 10.207 & 8.014 \\
\hline P8L2 & 17 & 6.040 & 8.244 & 10.220 & 8.133 \\
\hline P6L4 & 17 & 5.891 & 8.306 & 10.182 & 7.931 \\
\hline P4L6 & 17 & 4.963 & 8.011 & 9.424 & 7.283 \\
\hline P2L8 & 17 & 5.578 & 7.281 & 9.172 & 7.619 \\
\hline L10 & 17 & 7.043 & 7.761 & 10.481 & 7.682 \\
\hline
\end{tabular}

Table 3. Direct georeferencing accuracy of the ZY-3 TLC images in the dataset 2 .

\begin{tabular}{|c|c|r|r|r|r|}
\hline \multirow{2}{*}{ Layout } & Number & \multicolumn{4}{|c|}{ RMSE (m) } \\
\cline { 3 - 6 } & of ICPs & North & East & Planimetry & Height \\
\hline P0 & 9 & 782.375 & 1114.162 & 1361.422 & 340.731 \\
\hline P10 & 9 & 7.913 & 6.740 & 10.395 & 4.654 \\
\hline P8L2 & 9 & 6.744 & 6.666 & 9.482 & 4.951 \\
\hline P6L4 & 9 & 7.410 & 6.692 & 9.985 & 5.422 \\
\hline P4L6 & 9 & 7.013 & 6.161 & 9.335 & 5.484 \\
\hline P2L8 & 9 & 6.220 & 6.329 & 8.874 & 5.171 \\
\hline L10 & 9 & 7.185 & 6.377 & 9.607 & 5.000 \\
\hline
\end{tabular}

Table 4. Direct georeferencing accuracy of the ZY-3 TLC images in the dataset 3.

We can see from the results in Tables 3 and 4 that the CCDdetector look angles calibrated by combining GCPs and GCLs perform very well in the direct georeferencing of the ZY-3 TLC images. Due to the large biases between the laboratorycalibrated values of the orientation parameters and their in-orbit true values, the initial direct georeferencing accuracy of the ZY3 TLC images without the geometric calibration can only reach the kilometre level. Taking the CCD-detector look angles calibrated by using the layout P10 in the dataset 1 as input orientation parameters, the direct georeferencing accuracy of both the datasets 2 and 3 is significantly improved to better than $11 \mathrm{~m}$ in planimetry and $9 \mathrm{~m}$ in height. Meanwhile, according to the CCD-detector look angles calibrated by using the layouts P8L2, P6L4, P4L6, P2L8, and L10, the direct georeferencing accuracy of the ZY-3 TLC images is all almost the same as that achieved by using the layout P10. It demonstrates that substituting GCLs for GCPs in the geometric calibration has also almost no effect on the direct georeferencing accuracy.

It should be pointed out that when we perform the geometric calibration of the ZY-3 TLCs according to Equations 3, 7, and 8, the measurements of the satellite positions and attitudes and the measurements of the image-space and object-space coordinates of both the GCPs and GCLs in the dataset 1 are all considered free of errors. In fact, however, it is inevitable that these measurements have more or less errors. Consequently, these measurement errors are fully absorbed by the calibrated CCDdetector look angles, that is, the calibrated CCD-detector look angles have more or less errors. Besides, the measurements of the satellite positions and attitudes in the datasets 2 and 3 also have errors. Due to the comprehensive influences of the calibration errors and the measurement errors, the direct georeferencing accuracy of the ZY-3 TLC images in both the datasets 2 and 3 is still worse than the expected accuracy.
In order to eliminate the influences of the above errors and achieve the best georeferencing accuracy, the block adjustment with ground controls is preferred. For this reason, the rational function model with additional affine transformation parameters was used to perform the block adjustment of the ZY-3 TLC images in the datasets 2 and 3 . In the block adjustment, four GCPs denoted as $P_{1}$ to $P_{4}$ in Figures $2 \mathrm{~b}$ and $2 \mathrm{c}$ were used, and the remaining GCPs were considered as the ICPs. The block adjustment accuracy of the datasets 2 and 3 is listed in Tables 5 and 6 , respectively.

\begin{tabular}{|c|c|c|c|c|c|c|}
\hline \multirow{2}{*}{ Layout } & \multirow{2}{*}{$\begin{array}{c}\text { Number } \\
\text { of GCPs }\end{array}$} & Number & \multicolumn{5}{|c|}{ RMSE (m) } \\
\cline { 4 - 7 } & & & North & East & Planimetry & Height \\
\hline P10 & 4 & 13 & 1.484 & 1.370 & 2.019 & 2.192 \\
\hline P8L2 & 4 & 13 & 1.566 & 1.419 & 2.113 & 2.028 \\
\hline P6L4 & 4 & 13 & 1.644 & 1.610 & 2.302 & 1.912 \\
\hline P4L6 & 4 & 13 & 1.492 & 1.734 & 2.287 & 2.139 \\
\hline P2L8 & 4 & 13 & 1.869 & 1.596 & 2.457 & 2.112 \\
\hline L10 & 4 & 13 & 1.841 & 1.732 & 2.528 & 2.613 \\
\hline
\end{tabular}

Table 5. Block adjustment accuracy of the ZY-3 TLC images in the dataset 2 .

\begin{tabular}{|c|c|c|c|c|c|c|}
\hline \multirow{2}{*}{ Layout } & \multirow{2}{*}{$\begin{array}{c}\text { Number } \\
\text { of GCPs }\end{array}$} & Number & \multicolumn{4}{|c|}{ RMSE (m) } \\
\cline { 4 - 7 } & & 5 & North & East & Planimetry & Height \\
\hline P10 & 4 & 2.497 & 1.965 & 3.177 & 2.777 \\
\hline P8L2 & 4 & 5 & 0.886 & 2.032 & 2.216 & 2.559 \\
\hline P6L4 & 4 & 5 & 1.328 & 1.917 & 2.332 & 2.731 \\
\hline P4L6 & 4 & 5 & 0.968 & 2.400 & 2.588 & 2.526 \\
\hline P2L8 & 4 & 5 & 0.817 & 1.950 & 2.114 & 2.501 \\
\hline L10 & 4 & 5 & 2.513 & 1.794 & 3.087 & 2.021 \\
\hline
\end{tabular}

Table 6. Block adjustment accuracy of the ZY-3 TLC images in the dataset 3 .

In Tables 5 and 6 , the georeferencing accuracy of the ZY-3 TLC images is further improved after the block adjustment with four GCPs. For both the datasets, the block adjustment can achieve an accuracy of better than $3.5 \mathrm{~m}$ in planimetry and $3.0 \mathrm{~m}$ in height, no matter the input orientation parameters are calibrated by using the layouts P10, P8L2, P6L4, P4L6, P2L8, or L10. Meanwhile, the largest difference between these block adjustment accuracies is only $1.063 \mathrm{~m}$ in planimetry and $0.756 \mathrm{~m}$ in height, which is smaller than the measurement errors of the image-space coordinates of the GCPs. It further demonstrates that performing the geometric calibration of the ZY-3 TLCs combining GCPs and GCLs is feasible and effective. In the geometric calibration, substituting GCLs for GCPs has almost no effect on the block adjustment accuracy as well.

\section{CONCLUSIONS}

In this paper, a line-based geometric calibration model of the ZY-3 TLCs is established by using the coplanarity constraint that the projection line, the ground line, and the line determined by the projection centre and one end point of the ground line must always lie in a same plane. Based on the point-based and line-based geometric calibration models, a feasible geometric calibration approach for the ZY-3 TLCs combining GCPs and GCLs is presented.

The experimental results of three $\mathrm{ZY}-3$ datasets have shown that GCLs can also provide effective ground control information for the geometric calibration. Moreover, substituting GCLs for the absent GCPs has almost no effect on the calibration accuracy of the CCD-detector look angles, the direct georeferencing accuracy, and the block adjustment accuracy. Therefore, when 
sufficient evenly distributed GCPs are unavailable due to the lack of clear point features in the field or the large date interval between the ZY-3 TLC images and the reference DOM and DEM, performing the geometric calibration combining GCPs and GCLs can provide a better and more effective solution.

Of course, more ZY-3 datasets with much longer date interval are needed to evaluate the performance of the presented geometric calibration approach. Besides, the effects of the length, the direction, and the number of image points of GCLs on the geometric calibration also needs to be studied further.

\section{ACKNOWLEDGEMENT}

This work was supported by the National Basic Research Program of China (973 Program) (Grant No. 2012CB719902) and the China Postdoctoral Science Foundation (Grant No. 2014M562065).

\section{REFERENCES}

Cao, J., Yuan, X., and Gong, J., 2015. In-orbit geometric calibration and validation of ZY-3 three-line cameras based on CCD-detector look angles. Photogrammetric Record, 30(150), pp. 211-226.

Chen, Y., Xie, Z., Qiu, Z., Zhang, Q., and Hu, Z., 2015. Calibration and validation of ZY-3 optical sensors. IEEE Transactions on Geoscience and Remote Sensing, 53(8), pp. 4616-4626.

Crespi, M., Colosimo, G., Vendictis, L., Fratarcangeli, F., Pieralice, F., 2010. GeoEye-1: analysis of radiometric and geometric capability. Lecture Notes of the Institute for Compute Sciences, Social Informatics and Telecommunications Engineering, 43(7), pp. 354-369.

De Lussy, F., Greslou, D., Dechoz, C., Amberg, V., Delvit, J. M., Lebegue, L., Blanchet, G., and Fourest, S., 2012. PLEIADES HR in flight geometrical calibration: location and mapping of the focal plane. In: The International Archives of the Photogrammetry, Remote Sensing and Spatial Information Sciences, Melbourne, Australia, Vol. XXXIX, Part B1, pp. 519523.

Gachet, R., 2004. SPOT5 in-flight commission: inner orientation of HRG and HRS instruments. In: The International Archives of the Photogrammetry, Remote Sensing and Spatial Information Sciences, Istanbul, Turkey, Vol. XXXV, Part B1, pp. 535-539.

Grodecki, J., and Lute, J., 2005. IKONOS geometric calibration. In: Proceedings of the ASPRS 2005 Annual Conference, Baltimore, USA.
Habib, A. F., and Alruzouq, R. I., 2004. Line-based modified iterated hough transform for automatic registration of multisource imagery. Photogrammetric Record, 19(105), pp. 5-21.

Karjalainen, M., Hyyppä, J., and Kuittinen, R., 2006. Determination of exterior orientation using linear features from vector maps. Photogrammetric Record, 21(116), pp. 329-341.

Marcato Junior, J., and Tommaselli, A. M. G., 2013. Exterior orientation of CBERS-2B imagery using multi-feature control and orbital data. ISPRS Journal of Photogrammetry and Remote Sensing, 79, pp. 219-225.

Mulama, D., 2004. On-orbit geometric calibration of the OrbView-3 high resolution imaging satellite. In: The International Archives of the Photogrammetry, Remote Sensing and Spatial Information Sciences, Istanbul, Turkey, Vol. XXXV, Part B1, pp. 1-6.

Radhadevi, P. V., Müller, R., d'Angelo, P., and Reinartz, P., 2011. In-flight geometric calibration and orientation of ALOS/PRISM imagery with a generic sensor model. Photogrammetric Engineering \& Remote Sensing, 77(5), pp. 531-538.

Tommaselli, A. M. G., and Medeiros, N. G., 2010. Determination of the indirect orientation of orbital pushbroom images using control straight lines. Photogrammetric Record, 25(130), pp. 159-179.

Wang, M., Yang, B., Hu, F., and Zang, X., 2014. On-orbit geometric calibration model and its applications for highresolution optical satellite imagery. Remote Sensing, 6, pp. 4391-4408.

Zhang, G., Jiang, Y., Li, D., Huang, W., Pan, H., Tang, X., and Zhu, X., 2014. In-orbit geometric calibration and validation of ZY-3 linear array sensors. Photogrammetric Record, 29(145), pp. $68-88$

Zhang, Y., Hu, B., and Zhang, J., 2011. Relative orientation based on multiple conjugate features. ISPRS Journal of Photogrammetric and Remote Sensing, 66(5), pp. 700-707.

Zhang, Y., Zheng, M., Xiong, J., Lu, Y., and Xiong, X., 2014. On-orbit geometric calibration of ZY-3 three-line array imagery with multistrip data sets. IEEE Transactions on Geoscience and Remote Sensing, 52(1), pp. 224-234.

Zhang, Z., Zhang, Y., Zhang, J., and Zhang, H., 2008. Photogrammetric modeling of linear features with generalized point photogrammetry. Photogrammetric Engineering \& Remote Sensing, 74(9), pp. 1119-1127. 